\title{
TumTá and Pisada: Two Foot-controlled Digital Dance and Music Instruments Inspired by Popular Brazilian Traditions
}

\author{
João Tragtenberg ${ }^{12}$, Filipe Calegario ${ }^{12}$, Giordano Cabral $^{2}$, Geber Ramalho $^{2}$ \\ ${ }^{1}$ CIIMUS / Instituto SENAI de Inovação para TICs / SENAI-FIEPE \\ Rua Frei Cassimiro, 88 - Santo Amaro - 50.100-260 - Recife - PE \\ ${ }^{2}$ MusTIC / Centro de Informática / Universidade Federal de Pernambuco \\ Av. Jornalista Aníbal Fernandes, s/n - Cidade Universitária (Campus Recife) - 50.740-560 - Recife - PE \\ tragtenberg@gmail.com, fcacecin.ufpe.br \\ grecacin.ufpe.br, glracin.ufpe.br
}

\begin{abstract}
This paper presents the development process of “TumTá”, a wearable Digital Dance and Music Instrument that triggers sound samples from foot stomps and "Pisada," a dance-enabled MIDI pedalboard. It was developed between 2012 and 2017 for the use of Helder Vasconcelos, a dancer and musician formed by the traditions of Cavalo Marinho and Maracatu Rural from Pernambuco. The design of this instrument was inspired by traditional instruments like the Zabumba and by the gestural vocabulary from Cavalo Marinho, to make music and dance at the same time. The development process of this instrument is described in the three prototyping phases conducted by three approaches: building blocks, artisanal, and digital fabrication. The process of designing digital technology inspired by Brazilian traditions is analyzed, lessons learned, and future works are presented.
\end{abstract}

\section{Introduction}

"TumTá"1 is a wearable instrument in the form of a pair of insoles to be placed inside the shoes. The gestural design of the instrument was focused on the foot-stomping gesture with the heels. The mapping is straightforward, relating the stomping to the triggering of sound samples; the sound volume depends directly on the intensity of the stomp. The selection of the samples to be triggered can be done through an auxiliary interface called "Pisada", which consists of a series of pads distributed across the floor to be stepped on.

The development process has taught some lessons about the development of Digital Dance and Music Instruments (DDMI) [1, 2], by combining the expression through body and sound at the same time. Another exciting aspect of this process was the artistic context that guided its development. Instead of following aesthetic principles from European hegemonic cultures, a common bias in most of the music technology research and development, this process was based on grassroots popular Brazilian traditions [3]. This aspect has a lot to contribute to the Computer $\mathrm{Mu}-$ sic area and may be best suited for musicians and dancers based on similar traditions.

\footnotetext{
${ }^{1}$ Videos available on https://youtu.be/m4q6iD513pY and https: / /youtu.be/d0F54u015-w
}

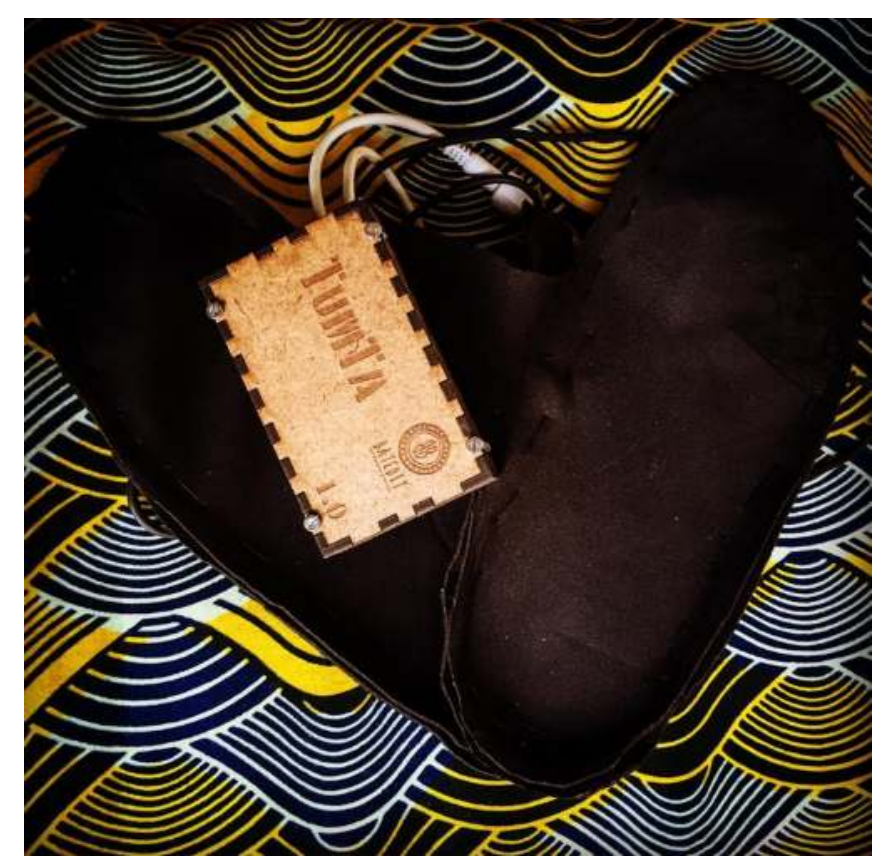

Figure 1: TumTá's insoles and transmitter hardware

\section{Motivation}

The "TumTá" started from a demand of the artist Helder Vasconcelos of an instrument that produced sound from dance gestures with a direct relation. The development of an instrument for music and dance was quite challenging. We found that the bibliography mostly differed between Digital Musical Instruments (DMIs) and Interactive Dance Systems (IDSs) and few references shared a perspective for expression through both art forms at the same time. The concept of Digital Instruments of Dance and Music (DDMIs) was created during this research process [1].

The development of "TumTá" began in 2012 and featured several stages of prototype development with frequent evaluation meetings with Helder Vasconcelos. The design process was focused on the use of Helder and guided by his artistic needs, without having been tested by any other user until 2017. This whole process happened without any funding, concomitantly with other works, and without a research laboratory infrastructure. 


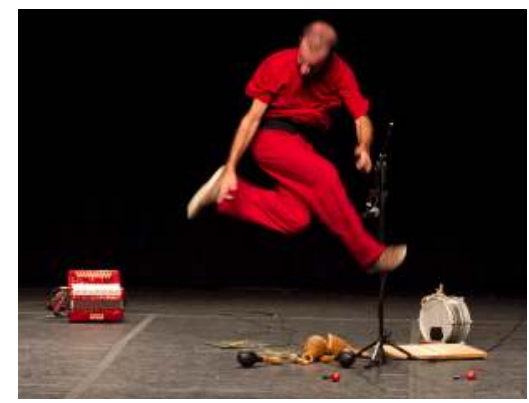

Figure 2: Helder Dancing/Playing “TumTá” during his performance

\subsection{Helder Vasconcelos}

Helder Vasconcelos is an artist renowned for his work as a musician, actor, and dancer. His formation took place in the brincadeiras of Cavalo Marinho (a famous theater from Pernambuco) and Maracatu Rural (tradition linked to the carnival of Pernambuco). He acted as a musician playing percussion instruments and eight-bass accordion in the band Mestre Ambrósio, where he also danced and performed characters during concerts. He also created two solo performances: "Espiral Brinquedo Meu," where the primary language was theater, and "Por Si Só," mainly driven by dance. In this second solo, he used Interactive Dance Systems created by Armando Menicacci ${ }^{2}$ to produce sounds and projections from his dance.

From the experience of working on another solo with digital technology, Helder already had a clear guideline for the technologies he was looking for: autonomy of use. During his second solo, the sensors and cameras he used to dance to interact in real time with several people were replaced by pre-recorded media because he couldn't use them by himself. He had also a specific demand for the functional requirements of his instrument and how he would make use of that of the device:

"I wanted very objectively a MIDI trigger on the heel that when I hit it on the floor it gave me an information, because all the rest I already had in my head [...] in a nutshell I wanted a deeper sound on the right foot and a sharper sound on the left foot, that was exactly the relation of the zabumba: the bacalhau ${ }^{3}$ in the left hand and, if you are right handed, and the mallet in the right hand. I wanted to reproduce exactly that on my right foot and on my left foot, which is precisely the zabumba's 'Tum' and the bacalhau's 'Tá'. So that's why the instrument ended up with that name, I wanted a 'Tum' on the right foot and a 'Ta' on the left foot. Although I already glimpsed several things "(Interview with Helder Vasconcelos)

This reference to the Zabumba, an instrument widespread in many genres of the North-eastern Brazilian music, shows how "TumTá" clearly presents an instru-

\footnotetext{
${ }^{2}$ Armando Menicacci is a research choreographer and professor of new media for interdisciplinary performance at Université di Quebec à Montréal (UQAM) and one of the first users of the "Isadora" programming environment.

${ }^{3}$ The Zabumba is a Brazilian percussion instrument that is played with a mallet and thin stick called bacalhau
}

mental inheritance[4]. The idea of an instrument that can trigger only two percussive sounds, one high-pitched and another low-pitched could be seen through hegemonic traditions as an instrument without much expressivity or diversity [5], but in the context of popular Brazilian music and many other traditions there are many instruments pervasive to many genres that are undoubtedly expressive and diverse with just two classes of sounds.

This search for expression both musical and corporal is a recurrent element in the trajectory of the artist and he credits his formation to those traditions, where this relationship is latent. Helder Vasconcelos systematized his learning process in these traditions in principles for making music, dance or theater. His principles are based on the idea that a gesture or a sound starts from a common essence, which he calls the generating impulses:

"The generating impulse of something that can become a dance, a song or a theater is the same. So I do not see it separately. It will all depend on the artistic necessity. What do I mean? What do I want to say? What do I want to build? [...] This creative impulse can become a character, it can become a choreography, or it can become a beat, a rhythm, or a song. [...] I don't see them as three integrated things or three separate things. In the place of the creative impulse I don't think what it will be." (Interview with Helder Vasconcelos)

From this theoretical background, Helder sought an instrument capable of revealing this common essence of gestures and sounds, and he was seeking for this instrument to instantly translate the impulsive quality of the impact of the footfall on the ground with the impulsiveness of the sound produced. Our challenge was to develop a device for perceiving in real-time the impulsiveness of a foot stomp and mapping it into sound.

\subsection{Artistic References}

As well as searching for an instrument with an instrumental inheritance of the Zabumba, the choice of this gesture of foot stomps with the heel had an inspiration in the dances of Cavalo Marinho, that many dance steps share this bold stomping:

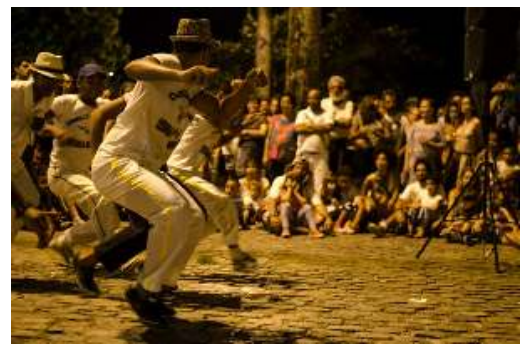

Figure 3: Totó Teles and Cavalo Marinho Estrela Brilhante from Condado(PE) - Brazil brincadores dancing a trupé

"This expression in Maracatu and even more in Cavalo Marinho, it is in the contact of the foot with the ground. We call it 'Trupé', the dance, the movement. It is a very percussive movement very naturally between the 
foot and the floor. So it comes from my school from the traditions that I participate in. This is already like this, so it was just a matter of empowering, saying, 'what if it actually made some sound out of that?"' (Interview with Helder Vasconcelos)

The steps of Cavalo Marinho have the characteristic of marking a rhythm very characteristic and common to several rhythms of Brazilian popular music. It is the same rhythmic cell of the baião, that usually is played by a zabumba:

"In several trupés of Cavalo Marinho we already do that [playing baião rhythms], even without making that sound, but we already play the zabumba thing. The Baião, the classic form of 'Tum Tum Tá Tum Tum' you play it with your foot, even without sound, dancing Cavalo Marinho. That's why it was so objective for me: 'If I have this, it will appear' "(Interview with Helder Vasconcelos)

This tight connection with a cultural tradition contributes to the degree of instrumentality [6] of "TumTá", allowing it to be perceived by musicians as a musical instrument and to create a pact with the audience [7] on understanding what sounds are connected to which gestures. These aspects are a rare situation for a new instrument, with which usually the audience does not know what to expect from the interaction. A dance with rhythmic characteristics of the foot contact with the ground strengthens the recognition of the dance made with the instrument and incorporates a gestural repertoire of this tradition. Several other tap dance traditions already carry an intimate relationship of percussive musical creation with footsteps, which brings to this mapping strategy an attractive potential for expressiveness.

\section{Similar Instruments}

\subsection{Miburi}

The Yamaha Miburi was an interactive whole-body wearable system with several sensors distributed throughout the body. Japan's Yamaha Experimental Division launched it in 1994. The system consists of a blouse with six bending sensors in the arm joints, two hand controllers, each with ten pressure-sensitive buttons and continuous controllers for the thumbs. The system also included two adjustable sized insoles with piezoelectric heel and toe sensors that detect tapping gestures. All these sensors were connected with a cable to a unit used as a belt. This unit communicated wirelessly (only for the version sold in Japan, for the exported versions only a cable connection was possible) with a sound synthesizer unit that had its amplifier.

\subsection{Expressive Footwear}

"Expressive Footwear" was developed by the Responsive Environments Group, coordinated by Joe Paradiso of the MIT Media Lab. Its interface consisted of a pair of snickers with 12 sensors that continuously send 16 raw data on the movement of the feet. It was developed to capture all the gestural possibilities of the feet like flexion, inclination, position in space in three dimensions, turns, orientation, pressure against the floor and foot stomps with the heel or tip of the foot.

The instrument was inspired directly by the Miburi's foot module during a visit of Joe Paradiso to the Yamaha experimental division. He claimed to have been inspired by its insole, seeking to overcome its limitation by adding sensors for continuous feet gesture recognition. The main guidelines were to eliminate the wires that connected the Miburi's insoles to the central unit, keeping all electronics wearable on the footwear. He sought a perception of continuous qualities of foot movement rather than discrete shots, which increased the possibility of interaction for free foot gestures and gestures of interaction with the ground [8].

\subsection{Other Similar Instruments}

Several other instruments have been developed for foot control beyond Miburi and Expressive Footwear. An example cited by Paradiso is a system with piezoelectric sensors for tap dancers [9]. Jônatas Manzolli developed a similar device in 1997 for the performance AtoContAto with the dancer Christiane Matallo [10].

\section{Development Process}

The development of "TumTá" started with the simplest and fastest solutions to trigger sounds from foot gestures. The prototypes were gradually evolving and acquiring increasing complexity and robustness. In order to overcome challenges, physical and functional prototypes were created to enable a practical evaluation of each solution proposition. The collaborative evaluation by the team brought up positive and negative points of each solution, and the discussion directed the approach we should take for the next prototype.

"Pisada" was developed to be an accessory for changing "TumTá" sample pairs, but it followed a parallel development process and ended up serving also as an independent Digital Dance and Music Instrument, serving for much more purposes. That is why we will present it in a separate section of this paper.

We started off the project with quick and dirty prototypes using off-the-shelf technology from game controllers (Nintendo Wiimote and Microsoft Kinect). Deciding on the approach of wearable sensors, we used an Arduino with some shields for connecting a DIY pressure sensor and an XBee transceiver to a Pure Data patch through an artisanal approach for the electronics and case. After validating the idea, in the next prototyping phase, we improved the robustness and size of the system using digital fabrication tools, designing a custom PCB and lasercut enclosure, a better transceiver and a more user-friendly software interface.

These three approaches using ready-made building blocks, hand-crafting, and with digital fabrication [11] have shown to be an exciting design process. We used each process' advantage in favor of each part of the process. These approaches were analyzed in the development 
of the Prosthetic Instruments, which showed some similar challenges we faced creating a new instrument for the specific performance of artists that were not the hardware developers.

In the subsections below, we described in further details the challenges and advantages we face on each part of the process.

\subsection{First Prototyping Phase: Building Blocks}

In the same meeting, some researchers from MusTIC research group, we used off-the-shelf building blocks prototypes that were assembled on the same day. Jerônimo Barbosa presented a prototype made with Microsoft Kinect where the quantity of movement of the whole body activated a state machine. This prototype did not have a direct relationship between gesture and sound, but a more abstract connection and was not connected to what Helder was seeking. The other prototype presented was with a Wiimote of the Nintendo Wii console by the second author. The control was taped to the dancer's ankle kand a simple mapping strategy with OSCulator ${ }^{4}$ was created to trigger MIDI notes when the sensor's accelerometer data passed a threshold. Despite the high latency and size of the controller, this prototype brought a positive reaction in Helder:

"Since the first test with the Wii tied with masking tape, it was very, very, very stimulating. I already felt something happening [...] that first test already had a stimulus to say "if it works I'll use"" (Interview with Helder Vasconcelos)

This Wiimote solution represents a widespread approach, having been one of the standard interfaces of the NIME community [12]. This first stage made clear the artist's choice for a wearable system, which was an essential constraint for development. We discarded the option of the camera-based solution because of many factors, for example, the need for lighting calibration, the possibility of occlusion, or the high latency of the cameras.

Even though the building blocks were not as small and with a fast reaction, it was enough to validate the idea, which leads us to improve the next prototypes in size, sensing accuracy and gesture-to-sound latency.

\subsection{Second Prototyping Phase: Artisanal}

Once established that the instrument would be a wearable device, we began to develop more responsive prototypes with a clear goal of having a lower latency. The first prototype craft was made with a pressure sensor (FSR 402 from Interlink) connected to a protoboard and an Arduino UNO. Every time the pressure went beyond a threshold, it triggered a MIDI note that in a DAW triggered a sound. We performed tests by tapping the sensor with a finger on a table and perceived outstanding responsiveness, without perceiving any latency.

Nevertheless, when we did the first tests with the sensors inside the shoes, the sensors broke on the first week

\footnotetext{
${ }^{4}$ https://osculator.net/
}



Figure 4: "TumTá's" second prototype made out of arduino uno with shields (to be enclosed in a travel soap case)

of tests due to the fragile structure of the sensors and heavy pressure load of the foot stomps. We also tried a piezo sensor, but no matter how much hot glue we put into protecting it, it always broke on the first stomps. The ruggedness of the wearable system, especially for dancing, is a great challenge for the hardware developer. Similar problems were found by the MIT Media Lab team while developing the Expressive Footwear: "A dancer's foot is indeed a hostile environment for sensitive electronics, and as we have been reminded repeatedly by experience, everything needs to be well attached or latched down - anything that can move will sooner-or-later break off" [8]

We migrated to a do-it-yourself sensor alternative with conductive foam that the XBee radio had come. This alternative was inspired by a tutorial published on the Instructables portal by Kyle McDonald [13]. This prototype showed a much less accurate reading than the purchased sensor and presented a very noisy signal, but it was very robust at impact.

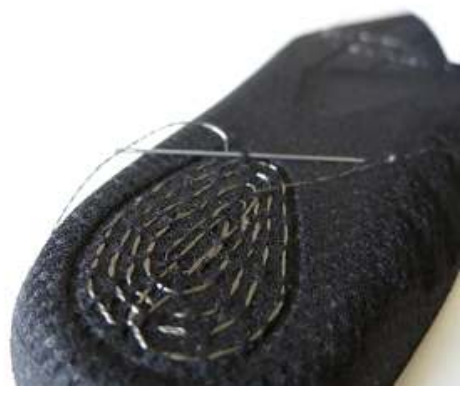

Figure 5: DIY sensor made out of a conductive foam insole and conductive thread electrodes

The next prototype was made from out of an antistatic insole made of conductive foam. We used conductive stainless steel (Bekinox VN 12 / 2X275 / 175S / 316L from Bekaert) for the electrodes, which presented an excellent resistance to impact. Sensors previously created with small pieces of conductive foam had the problem of sliding during use, a problem solved when the sensor was shaped as an insole by fitting perfectly inside the shoe. The whole stack Arduino Shields ${ }^{5}$ was enclosed inside a plastic trav-

\footnotetext{
${ }^{5}$ This artisanal approach also used Arduino Shields building blocks, instead of using custom soldered breadboards, this accelerated a lot the hardware development process
} 
eler's soap-dish, fixed with an elastic bandage around the waist.

Another challenge for the development of wearable instruments that we found refers to the electrical noise of the human skin. We noticed a noise in the sensor signals when the foot came in contact with the sensor. It was necessary to coat the insole with a rubber (neoprene) to isolate the circuit of this noise. Any solution with a conductive line, lacking electrical insulation, is subject to these noises.

Even with several problems in these first prototypes, it gave a much better solution than the taped Wiimote, allowing him to give his first presentation to a broad audience at "Mostra Rumos" in São Paulo's Itaú Cultural main theater, part of the Rumos Itaú Cultural dance award for 2012-2014. The artisanal solution did not allow an autonomous use, needing of a specialized professional to fix software and hardware bugs which were still frequent. This approach was very customizable but gave unstable solutions that could be improved in ruggedness, size, and userfriendliness.

\subsection{Third Prototyping Phase: Digital Fabrication}

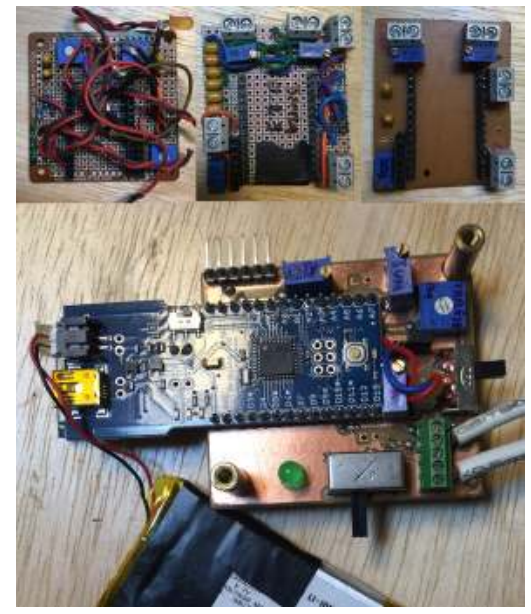

Figure 6: Sucessive versions of the prototype's $P C B$, improving it's quality and reducing the amount of wires

With the arrival of Fablab in the city of Recife, we had access to a laser cutter, with which we were able to take advantage of techniques of digital fabrication. This approach enabled us to create prettier and better enclosures with greater precision and robustness. We also started manufacturing our PCBs with the laser-cutter, which lead to much more rugged circuits that did not present bad contact issues from fatigued wires. The new circuits went through several stages of development (Figure 6), seeking a smaller size and a more trustworthy circuit with fewer wires.

We also had several problems with the wireless transmission. The XBee s1 module had a good range of places without many $2.4 \mathrm{GHz}$ devices (Mainly Wifi and Bluetooth devices), but as the noise grew, the shorter was the range it worked. For a fast solution, we changed to an equivalent more powerful transmitter (XBee Pro S1) to have a strong signal even in electromagnetically noisy places. Packet loss was somewhat inadmissible for the low fault tolerance that the condition of artistic presentation demands.

The stomping recognition used an algorithm that the first step was responsible to store the sensor data in a sliding window with the last five readings. A median filter averaged these five values to reduce noise from the sensors. From the last five averages, a derivative approximation of the data was calculated with a backward finite difference algorithm. The values of the derivatives were monitored through a Noise Gate filter, only to be considered when they passed a certain threshold. When a maximum local value was found, an event was triggered, and that value was related to the intensity of the stomp. As the data presented at least two peaks for each stomp, we implemented a debounce algorithm to only consider the first one in a time interval of $150 \mathrm{~ms}$. This interval was much bigger than the regular interval between bounced peaks, but since it was not possible to give two consecutive stomps in a time shorter than that we kept the highest stability of the instrument.

The use of the pressure's rate of variation (numerical derivative) to detect the stomps allowed them to be differentiated from simple steps. When walking, the pressure ranges from minimum to maximum values as the weight is transferred from one foot to the other, but the pressure variation in each foot occurs very slowly. The detection threshold allowed to incorporate the range in which the derivative of the pressure corresponded to basic steps, allowing the user to walk without triggering any sound. The stomps occur with a higher pressure rate variation, and the adjustment of this threshold was made with tests of walking steps against stomps.

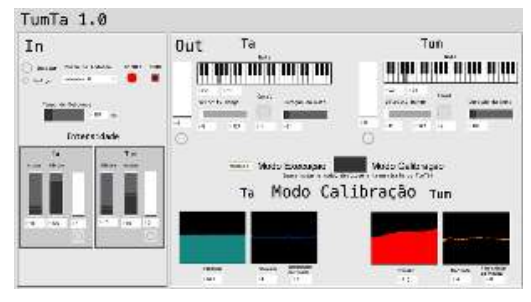

Figure 7: Max/MSP patch for mapping the sensor data into MIDI note triggers

For improving the usability, a Max/MSP patch was developed with an improved graphical user interface, allowing Helder to quickly understand how to calibrate his instrument and configure simple parameters like the dynamic range and MIDI notes and channels each foot would trigger.

After almost three years of development, we came to a stable version of "TumTá". With this version, Helder Vasconcelos used it in the debut of his third solo "Eu Sou." It was possible to have a dynamics control between notes of weak, medium, or strong intensities with few nuances between them. The solo debut took place at the SESC Pompéia theater in São Paulo as part of an occupation 
for a month, where the artist also presented his other two solos. The dance and music made throughout the show with "TumTá" allowed much freedom for improvisation and control over the execution.

\subsection{Future challenges}

The main technical problem during development was related to the UART serial protocol used to read data in Max/MSP from the XBee Receiver. After the last version, we noticed that the standard UART baud rates $(9600$, 19200, 38400, 57600, 74880, 115200 bps) have a considerable amount of dropped packets. This issue is not related to regular devices, but to musical applications they are inadmissible. The low priority of the FTDI $^{6}$ driver reading USB serial data in the operating system of the computer may have also contributed in no small latency, jitter, and unrecognized stomps.

Recent studies have shown how solutions with Arduino communicating over serial ports with Max/MSP are unable to meet the high latency and jitter requirements of percussive musical instruments [14]. The authors suggest that the MIDI protocol should be used whenever possible. Even though the baud rate of 31250 bps is relatively low, it synchronizes with the microcontroller's clock value and does not drop any packet. It adds to that reason that the MIDI device USB drivers are optimized for a higher priority in the OS, considering its time sensitiveness. The common critiques around the limitation of MIDI messages to be limited to 7 bits (from 0 to 127 ) are not precise since the MIDI protocol can use Non-registered parameter Numbers (NRPN), system Exclusive messages (SysEx) or other methods to send continuous data with a more extensive bit range.

Some even more recent research shows that Open Sound Control (OSC) and MIDI Over Bluetooth Low Energy (BLE) protocols with the readily available ESP32 microcontroller can deliver data with a latency below the $10 \mathrm{~ms}$ accepted standard without the need of a hardware receiver [15].

Due to the artisanal nature of this sensor, it is hard to replicate it into similar products. The lack of sensitivity of the instrument for weaker foot stomps was a challenge that remained unsolved in this version because of the noisy signal from the sensor and represented the most noticeable problem from the users that tested it.

"Its sensitivity is so important [...] today, it is hard, he demands a lot. [...] it requires a certain posture and a certain pressure, a certain force for it to trigger the sound. [...] so it still has this tension [...] not triggering is very frustrating. It disorganizes a lot. It is as if you are going to play a note, you are going to play chord a piano and it didn't trigger any sound. [...] making it more sensitive, more precise in this way, that would also let me loosen up, it would increase the possibilities, because I would not be in this place of tension: 'Jeez, maybe it won't trigger anything" (Interview with Helder Vasconcelos)

\footnotetext{
${ }^{6}$ chip used to convert RS-232 serial protocol to USB
}

Another factor to be rethought is the need for a computer to operate the instrument; that is, we want the next versions to have a hardware sound unit to trigger the sounds. This factor would facilitate its use and logistics, allowing presentations to informal contexts and alternative places like squares, streets, etc. Currently, in addition to relying on the computer, it requires specific software that may be deprecated in future versions of the OS. It needs to have hardware that can have a $1 / 4$ " jack for triggering its samples and a USB and DIN MIDI output connector for more excellent compatibility with low latency with other gear.

\section{Pisada}

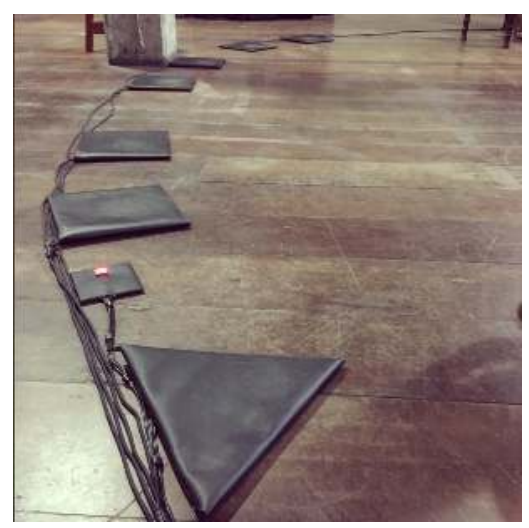

Figure 8: "Pisada" pads spread on the floor

"Pisada" is a DDMI designed initially for changing the sound banks of "TumTá" while dancing. Its sensor interface consists of ten square pads of $25 \times 25 \mathrm{~cm}$ connected to a hub through, to be pressed with the feet. It holds the same functional principle as a regular MIDI pedalboard. The main difference is the size and structure of the buttons, that are larger and spreadable around the stage, imposing fewer restrictions to body movement while pressing it.

Since it is a MIDI controller with ten buttons and ten pages that change the function of each pad, it can serve for much more than a "TumTá" accessory. It allows several musical controls in DAWs and Synthesizers such as turning on and off effects, triggering sounds or selecting tones.

A traditional MIDI pedalboard usually has several buttons separated by a few centimeters from each other, requiring the user to stand in a region of space and make a slow and careful movement to push each button. The "Pisada" allows the control gesture to be anywhere on the stage and pressed during a dance without a cognitive restriction on the performer's movement.

The "Pisada" works as an incredibly safe, accurate and straightforward to use position sensor. It can detect a region with a radius of up to $10 \mathrm{~m}$, by the length of its wires (which can be extended). Unlike computer vision techniques that require complicated procedures for adjusting brightness and configuring the division of space, 
"Pisada" can detect when the artist is in some position with effortless installation and use procedure. By having tactile feedback and being a visible object, it allows much more precise temporal control than camera position detection systems.

Beyond the initial function to alter the samples triggered by "TumTá", Helder Vasconcelos used it in his show "Eu Sou" in various ways like triggering playbacks for songs, activating and deactivated effects on his microphone, for recording live loops, triggering live recorded loops and activating or altering functions of other digital instruments like a tap-dancing floorboard with Tet Music's "Pulse Controller" 7. "Pisada" was the instrument that placed the controls which are usually on a backstage technician's hands, on the artist's feet.

It was made in an artisanal way with two galvanized iron sheets with rubber tabs on the corners. When it was pressed, the plates touch and close a contact measured by the microcontroller. When stepping it sends a note on signal and release sends a note off signal. There is only a simple debounce algorithm with a relatively high value of 300 milliseconds to ensure that a button is pressed once. They are all covered with black linoleum to be discreet on traditional stages.

In addition to the ten squared pads, two triangular pads are responsible for changing the button-to-MIDIevents mapping, in a metaphor of pages. The ten pages are shown in a seven segments LED display, allowing 100 different notes to be triggered. There is also visual feedback LED on each rectangular pad that lights up when pressed. The ten pads are connected to a central hub by long RJ45 cables of 10 meters, and this hub can be connected to a computer by a MIDI USB cable.

\subsection{Future challenges}

The instrument's simplicity made it very easy to use, but the artisanal process of building it takes too much time and is too much expensive since it uses thick iron sheets. It was also too heavy and presented serious airplane shipping problems. The cables represent a problem because they need to be dispersed on stage, and the performer cannot step on it, only allowing them to be positioned on the edges of the used stage area.

The instrument would benefit a lot by a changing in the material and structure to a lighter and more straightforward to make a structure and a robust wireless connection would surely be a much better alternative. Long cables are costly, with reasonably short durability and require much time to be positioned in space. It may also be interesting to add sensitivity to the pressure to afford new control possibilities.

The "Pisada" is a simple instrument developed with the premise of bodily expressiveness for precise musical control. It was designed to be robust, easy to learn, to have a low latency and precise responsiveness. It presents

${ }^{7}$ http://tetmusic.com/ a straightforward, low cost and robust solution for the detection of position in space.

\section{Sound Design}

The sound design of "TumTá" was all made in Ableton Live using its "Simpler" sampler instrument. The sampling technique was used for the simplicity and precision to trigger sounds from acoustic instruments from popular traditions and other electronic sound samples. Helder Vasconcelos triggered in his home studio his Bombos (an instrument similar to a zabumba used in his Boi Marinho tradition), Preacas (instrument from the tradition of Caboclinho, which consists of a percussive bow and arrow), a Kaïamb (a shaker from Réunion Island). Some synthesizer samples were also played and also samples of his voice.

The samples were recorded both as one-shot sounds and parts of a loop playing a baião rhythm with the Bombo. In some sample banks, both feet were mapped to the same one-shot samples. On other banks, one foot was mapped to the bacalhau stick of the bombo, giving a high pitched sound, while the other foot was mapped to a part of a loop that needed to be retriggered in precise time to keep the rhythm going in the beat. Specific dance steps made rhythm variations retriggering the sample in shorter periods. The kayamb was triggered in a looped pattern with a sizeable decaying amplitude.

It was mapped in a way that each new "Pisada" pad pressed changed the samples. The group of samples that played loops were built in a way that new banks repeated the last samples with new layers added to it, in a similar way to the structure of Electronic Dance Music, allowing him to give more liveness to a performance that a building up rhythm with superposed layers of sound timbres.

\subsection{Future challenges}

The versatility of a sampler was the right choice for the straightforward sound design based on traditions that rely only on acoustic instruments. A physical modeling synthesis would also be an exciting approach, giving more subtlety in random sound variations, typical from percussive acoustic instruments, but would lead to much more time and specific knowledge to be developed. This approach of allowing sound variations depending on velocity and on random parameters could also be used in the "TumTá" sampling engine with many samples per foot, and that would undoubtedly make the instrument a lot more expressive. The sampler also gives the instrument an exciting potential for the instrument to be adopted by other musicians and dancers, for the simplicity of customization and sharing of sound banks.

In literature, Sergi Jordà presents the concept of Diversity, that helps to define the concept of Music Instrument Efficiency [5]. The instrument's diversity is subcategorized in Micro-diversity, related to performance nuances (how two performances of the same piece can differ), Middiversity, related to performances' contrasts (how distinct 
two pieces played with the same instrument can be) and Macro-diversity related to stylistic flexibility (how the instrument adapts to different contexts). From this perspective, "TumTá" could easily be considered a not very diverse instrument, from a simplistic perspective that it needs to have many controls to be diverse.

"TumTá" was designed to be as diverse as a Zabumba, that even though it can trigger small variations of two sounds, it has a high diversity in all dimensions. The current lack of diversity of "TumTá" is related to its low dynamic range of control, only detecting foot stomps that are too hard. An improvement in the sensor interface and better sound design with a more expressive sampler would improve a lot its diversity and therefore, its efficiency.

\section{Conclusions}

This paper presents "TumTá" and "Pisada." Two original Digital Dance and Music Instruments inspired by Brazilian popular traditions. This process pointed out was how music and dance traditions could influence the development of new technology and is a rich source of inspiration for the design of original NIMEs. The development process of the instrument was described in technical details, following three main prototyping phases that overlapped each other, but each had a specific approach on Building Blocks, Artisanal, and on Digital Fabrication. Both future instruments challenges were presented separately and had a lot to improve, but are already used in professional artistic contexts with success.

\section{Lessons Learned}

From the development process, many technical lessons were learned from specific challenges in each prototyping phase:

- Building Blocks are a good approach for a first prototyping phase. Even though they are not customizable, it gives quick answers to major decisions.

- The Artisanal Approach is interesting for a highly customizable solution, giving freedom to have original ideas while is time-consuming, not easily reproducible and can lack reliability, precision, or ruggedness.

- Digital Fabrication techniques can shorten a lot the design process giving precise and reliable solutions with highly customizable prototypes. Nevertheless, the possibilities are a lot limited by the machine's constraints.

- Wearable devices for dancers need to be EXTRA RUGGED, and needs isolation because of the sweat.

- Wireless transmissions need to be powerful for long distances (beyond the noise around you)

- MIDI and OSC are always better solutions than custom serial protocols; there is no need for reinventing the wheel.

- Sample-based synthesis is great for percussive instruments and an accessible way to create sounds with a Brazilian musical identity.

\section{References}

[1] João Tragtenberg, Filipe Calegario, Giordano Cabral, and Geber Ramalho. Towards the Concept of Digital Dance and Music Instrument. In Proceedings of the International Conference on New Interfaces for Musical Expression, Porto Alegre, Brazil, 2019.

[2] João Tragtenberg. Instrumentos Digitais de Dança e Música: Uma Proposta de Paradigma para Potencializar o Design de Instrumentos para Expressão Musical e Corporal MSc. Thesis UFPE, 2017.

[3] Jerônimo Barbosa, Filipe Calegario, João Tragtenberg, Giordano Cabral, Geber Ramalho, and Marcelo M Wanderley. Designing DMIs for Popular Music in the Brazilian Northeast : Lessons Learned. Proceedings of the International Conference on New Interfaces for Musical Expression, pages 277-280, 2015.

[4] Filipe Calegario. Designing Digital Musical Instruments Using Probatio: A Physical Prototyping Toolkit. Computational Synthesis and Creative Systems. Springer International Publishing, Cham, 2019.

[5] Sergi Jordà. Digital Lutherie Crafting musical computers for new musics' performance and improvisation. Departament de Tecnologia, 26(3):531, 2005.

[6] Sarah-Indriyati Hardjowirogo. Instrumentality. On the Construction of Instrumental Identity. In Musical Instruments in the 21st Century Identities, Configurations, Practices. Springer, Berlin, 2017.

[7] Wayne Siegel. Dancing the Music: Interactive Dance and Music. In Roger T. Dean, editor, The Oxford Handbook of Computer Music, chapter 10, pages 191 - 213. Oxford University Press, Oxford, 2009.

[8] Joseph A. Paradiso. Footnotes: Personal reflections on the development of instrumented dance shoes and their musical applications. In in Quinz, E., ed., Digital Performance, Anomalie, digital arts, pages $34--49,2000$.

[9] A. Di Perna. Tapping into MIDI. Keyboard Magazine, page 27, 1988.

[10] Jônatas Manzolli, A. Moroni, and C. Matallo. AtoContAto: new media performance for video and interactive tap shoes music. In AtoContAto: new media performance for video and interactive tap shoes music., page 31, Bristol, 1998.

[11] Ian Hattwick, Joseph Malloch, and Marcelo Wanderley. Forming Shapes to Bodies: Design for Manufacturing in the Prosthetic Instruments. Proceedings of the International Conference on New Interfaces for Musical Expression, (August 2016):443-448, 2014.

[12] Kristian Nymoen. MuMYO - Evaluating and Exploring the MYO Armband for Musical Interaction. In Proceedings of the International Conference on New Interfaces for Musical Expression, pages 215-218, Bâton Rouge, US, 2015.

[13] Kyle McDonald. DIY Force Sensitive Resistor (FSR): https://www.instructables.com/id/DIY-Force-SensitiveResistor-FSR/, 2008.

[14] Andrew P McPherson, Robert H Jack, and Giulio Moro. Action-Sound Latency: Are Our Tools Fast Enough? Proceedings of the International Conference on New Interfaces for Musical Expression, 16:20-25, 2016.

[15] Johnty Wang, Axel Mulder, and Marcelo Wanderley. Practical Considerations for MIDI Over Bluetooth Low Energy as a Wireless Interface. In Proceedings of the International Conference on New Interfaces for Musical Expression, Porto Alegre, Brazil, 2019. 